\title{
Hamiltonian two-body system in special relativity
}

\author{
Philippe Droz-Vincent \\ LUTH \\ Meudon *
}

\begin{abstract}
We consider an isolated system made of two pointlike bodies interacting at a distance in the nonradiative approximation. Our framework is the covariant and a priori Hamiltonian formalism of "predictive relativistic mechanics", founded on the equal-time condition. The center of mass is rather a center of energy. Individual energies are separately conserved and the meaning of their positivity is discussed in terms of world-lines. Several results derived decades ago under restrictive assumptions are extended to the general case. Relative motion has a structure similar to that of a nonrelativistic one-body motion in a stationnary external potential, but its evolution parameter is generally not a linear function of the center-of-mass time, unless the relative motion is circular (in this latter case the motion is periodic in the center-of-mass time). Finally the case of an extreme mass ratio is investigated. When this ratio tends to zero the heavy body coincides with the center of mass provided that a certain first integral, related to the binding energy, is not too large.
\end{abstract}

\section{Introduction}

Classical relativistic dynamics of pointlike bodies has a long story; without claiming to be exhaustive let us mention the Wheeler-Feynman

* Observatoire de Paris, CNRS, Université Paris Diderot, 5 place Jules Janssen, 92195 Meudon, France 
(WF) electrodynamics [1] based upon the Fokker action [2, the three forms of dynamics (front form, point form, instant form) advocated by Dirac [3], and after the discovery of a famous No-Interaction theorem [4], various efforts made in order to circumvent it; for instance Predictive Mechanics [5] 6], the Singular Lagrangian method [8] and Constraint Dynamics [9]. In the last decade there were a few papers along the lines of WF [10] and also the work carried out by Lusanna et al. [11] in order to give a covariant status to the instant form.

Beside several $n$-body generalizations, most progresses have been devoted on the two-body problem, which is our present subject.

A first point was the possibility of actually having (unlike WF electrodynamics) second-order differential equations describing the motion of a finite number of degrees of freedom interacting at a distance. According to this view, the field that carries interaction is supposed to be eliminated, the space of initial data has a finite number of dimensions and a point in this space uniquely determines the subsequent motion [5] 6].

A second point was about a Hamiltonian formalism. We have in mind conservative mechanics: radiative corrections are neglected and we focus on isolated systems, characterized by a finite number of degrees of freedom and by Poincaré invariance. Under these conditions the nointeraction theorem [4] excludes the possibility of demanding that the physical positions be canonical throughout phase space (as was always done in classical mechanics). Relaxing this requirement implies an arbitrariness which can be removed by imposing a relationship between physical positions and canonical variables on some submanifold $\Sigma$.

In the a priori Hamiltonian approach of predictive mechanics [7, manifest covariance is realized with help of considering degrees of freedom that are geometrically natural but redundant (if compared to the classical situation). Positions and momenta are four-component objects, phase space has sixteen independent dimensions and we employ a multitime formalism (in a different spirit Todorov proposed to focus on the physical degrees of freedom only, in another formulation of dynamics which is covariant as well but includes first-class constraints [9]).

After discussing other possibilities, we put forward a natural prescription: physical and canonical positions must coincide when both physical positions are simultaneous with respect to the rest frame of the system [7]. In this approach a submanifold $\Sigma$ is obtained by selecting the configurations where the physical coordinate times $x_{1}^{0}, x_{2}^{0}$ are equal in the center-of-mass frame. An advantage of this equal-time prescription is to permit the contact with the constraint formalism, as 
shown in detail by L. Lusanna [12.

The two-body problem immediately suggests these important issues: center of mass and relative motion. In this article we intend to study their properties in the context of relativistic dynamics, aiming at a method in order to simplify the determination of the world lines.

A few exploratory results that we derived in the past [13] [14] have remained fragmentary, as most of them have been obtained with help of restrictive assumptions concerning the shape of the interaction potential. The present work is free of such limitation, aiming at the possibility of dealing with realistic interactions; such interactions cannot offer the simplicity of the academic models we considered long time ago: see for instance the unavoidable $P^{2}$ - dependence of an electromagnetic twobody potential proposed by Jallouli and Sazdjian [20], in the framework of relativistic quantum mechanics.

Before we focus on its equal-time version, we shall sketch the main lines of the a priori Hamiltonian approach in general.

In the next section we present the isolated two-body systems and introduce the center of mass.

In the rest of the paper we consider unipotential models (for these systems the individual energies are separately conserved).

In Section 3 we study the evolution of the canonical variables, irrespective of their relationship to physical positions. Later, in Section 4, we focus on the equal-time prescription and discuss a strategy for the determination of world-lines; the importance of circular orbits is emphasized. In 5 we consider the case of an extreme mass ratio; a toy model is presented in Section 6, and Section 7 is devoted to concluding remarks.

The velocity of light is taken as unity, except in the Appendix. When no confusion is possible, tensor indices are omitted and the contraction dot is employed also for tensors, for instance $J \cdot P$ stands for the vector $J^{\alpha \mu} P_{\mu}$.

\subsection{The a priori Hamiltonian formalism}

The canonical coordinates are $q_{a}^{\alpha}, p_{b \beta}$ in the sixteen-dimensional phase space; $q_{1}, q_{2}$ are points in Minkowski space $\mathcal{M}$ and $p_{1}, p_{2}$ are fourvectors.

The symplectic form $d q_{1}^{\alpha} \wedge d p_{1 \alpha}+d q_{2}^{\alpha} \wedge d p_{2 \alpha}$ corresponds to the Poisson brackets

$$
\left\{q_{a}^{\alpha}, p_{b \beta}\right\}=\delta_{a b} \delta_{\beta}^{\alpha}
$$


Note that the particle labels $a, b, c$ are not summed over when repeated. The Hamiltonian equations of motion

$$
\frac{\partial q_{a}}{\partial \tau_{b}}=\left\{q_{a}, H_{b}\right\} \quad \frac{\partial p_{a}}{\partial \tau_{b}}=\left\{p_{a}, H_{b}\right\}
$$

involve two Hamiltonian generators $H_{1}, H_{2}$ submitted to the predictivity condition

$$
\left\{H_{1}, H_{2}\right\}=0
$$

The two evolution parameters $\tau_{1}, \tau_{2}$ are suitable generalizations of the proper times (normalized to the masses). Our notation is choosen such that the generators can be identified as the half-squared masses; this allows to consider the masses as first integrals, redeeming the fact that phase space has redundant degrees of freedom.

For instance, in the trivial case of two free particles one is left with $2 H_{a}=p_{a}^{2}$, the physical positions reduce to $x_{a}=q_{a}$ and the evolution parameters are just $\tau_{a}=s_{a} / m_{a}$, where $s_{1}, s_{2}$ are the arc lenghts.

For interacting particles the Hamiltonians involve additional terms $V_{1}, V_{2}$ referred to as "potentials". In general $q_{1}, q_{2}$ differ from the physical positions, in fact $x_{1}, x_{2}$ are determined by the partial differential equations

$$
\left\{x_{a}, H_{b}\right\}=0 \quad \text { for } \quad a \neq b
$$

and some reasonable initial data. Solving these equations provides a correspondance between physical and canonical coordinates, say

$$
x, v \longleftrightarrow q, p
$$

which, inserted into the solutions of system (2), ensures that $x_{1}$ is a function of $\tau_{1}$ only and $x_{2}$ is a function of $\tau_{2}$ only, in other words (3) ensures the existance of one-dimensional world-lines [7]. This point is easily checked by introducing on phase space the Hamiltonian vector fields $X_{1}, X_{2}$ defined by

$$
X_{a} f=\frac{\partial f}{\partial \tau_{a}}=\left\{f, H_{a}\right\}
$$

for all phase-space function $f$. The predictivity condition corresponds to the vanishing of the Lie bracket $\left[X_{1}, X_{2}\right]$. Although the analytic shape of the Hamiltonians yields some information about first integrals, symmetries, etc, the physical meaning is fixed only once a solution to (3) has been specified. At this stage phase space is identified with the bundle product $T(\mathcal{M}) \times T(\mathcal{M})$.

Notation. $\left(H_{a}\right)_{\text {free }}=\frac{1}{2} p_{a}^{2}$, they generate $\left(X_{a}\right)_{\text {free }}$. 
When fixing numerical values $\frac{1}{2} m_{1}^{2}$ and $\frac{1}{2} m_{2}^{2}$ to the Hamiltonian generators, it is convenient to set

$$
\mu=\frac{1}{2}\left(m_{1}^{2}+m_{2}^{2}\right), \quad \nu=\frac{1}{2}\left(m_{1}^{2}-m_{2}^{2}\right)
$$

which amounts to

$$
m_{1} m_{2}=\sqrt{\mu^{2}-\nu^{2}}, \quad\left(m_{1}+m_{2}\right)^{2}=2 \mu+2 \sqrt{\mu^{2}-\nu^{2}}
$$

\section{Isolated two-body systems}

The Lie algebra of the Poincaré group is spanned by $P_{\alpha}, J_{\mu \nu}$. We separate external from internal variables by setting

$$
\begin{aligned}
& P=p_{1}+p_{2} \quad Q=\frac{1}{2}\left(q_{1}+q_{2}\right) \\
& y=\frac{1}{2}\left(p_{1}-p_{2}\right) \quad z=q_{1}-q_{2}
\end{aligned}
$$

Remark in general $Q$ is not the center of mass 1 .

Other definitions of $Q$ and $y$ (as conjugate to $\mathrm{P}$ and z respectively) were possible, but the advantage of ours is that it does not require to a priori fixthe numerical values of the constituent masses.

The standard Poisson brackets that do not vanish can be re-arranged as follows

$$
\left\{Q^{\alpha}, P_{\beta}\right\}=\left\{z^{\alpha}, y_{\beta}\right\}=\delta_{\beta}^{\alpha}
$$

and we can write

$$
J=Q \wedge P+z \wedge y=q_{1} \wedge p_{1}+q_{2} \wedge p_{2}
$$

Naturally $H_{1}, H_{2}$ are supposed to be Poincaré invariant (vanishing Poisson brackets with $P$ and $J$ ) and the initial conditions for solving (3) must be invariant as well.

From (9) we can derive several useful formulas; let us list some of them. Of course $\left\{Q^{\mu}, P^{2}\right\}=2 P^{\mu}$ and

$$
\left\{Q^{\mu}, P_{\alpha} P_{\beta}\right\}=\delta_{\alpha}^{\mu} P_{\beta}+P_{\alpha} \delta_{\beta}^{\mu}
$$

so if we define the projector orthogonal to $P$

$$
\Pi=\eta-\frac{P \otimes P}{P^{2}}
$$

\footnotetext{
${ }^{1}$ even for free particles it is center of mass only when the masses are equal.
} 
we get

$$
\left\{Q^{\mu}, \widetilde{z}^{2}\right\}=-2 \widetilde{z}^{\mu} \frac{P \cdot z}{P^{2}}
$$

with this notation that the tilde means an application of $\Pi$, say

$$
\widetilde{\xi}^{\alpha}=\Pi^{\alpha \beta} \xi_{\beta}, \quad \forall \xi
$$

the r.h.s. of (11) is orthogonal to $P$; it follows that

$$
\left\{Q \cdot P, \widetilde{z}^{2}\right\}=0
$$

On the other hand we compute

$$
\left\{Q \cdot P, \frac{P \otimes P}{P^{2}}\right\}=0
$$

in other words $Q \cdot P$ has a vanishing Poisson bracket with the projector П. Moreover we easily check that

$$
\left\{Q \cdot P, \frac{(y \cdot P)^{2}}{P^{2}}\right\}=0
$$

It is obvious that $\left\{Q \cdot P, y^{2}\right\}$ vanishes and we can apply the above formula to the identity

$$
\widetilde{y}^{2}=y^{2}-\frac{(y \cdot P)^{2}}{P^{2}}
$$

which yields

$$
\left\{Q \cdot P, \widetilde{y}^{2}\right\}=0
$$

For the relative variables note that the spatial piece of the one has a vanishing bracket with the time piece of the other (its conjugate):

$$
\{\widetilde{z}, y \cdot P\}=\{\widetilde{y}, z \cdot P\}=0
$$

Similarly it stems from (13) that

$$
\left\{Q \cdot P, \widetilde{z}^{\alpha}\right\}=\left\{Q \cdot P, \widetilde{y}^{\alpha}\right\}=0
$$

Of course we already know ten first integrals, namely $P_{\alpha}, J_{\mu \nu}$. We obviously have that

$$
\frac{\widetilde{P \cdot J}}{P^{2}}=\frac{z \cdot P}{P^{2}} \widetilde{y}-\widetilde{Q}-\frac{y \cdot P}{P^{2}} \widetilde{z}
$$




\subsection{Center of Mass}

The possibility to define a center of mass, using linear and angular momenta as basic ingredients, has been known long time ago [16.

In a previous work [15] we proposed this canonical definition for the components of the center of mass

$$
\Xi=\frac{J \cdot P}{P^{2}}+\left(\frac{P \cdot Q}{P^{2}}\right) P
$$

or equivalently

$$
\Xi=Q+\left(\frac{y \cdot P}{P^{2}}\right) z-\left(\frac{z \cdot P}{P^{2}}\right) y
$$

This can be transformed again, if we notice that

$P^{2} / 2 \pm y \cdot P=P \cdot p_{1},\left(\right.$ resp. $\left.P \cdot p_{2}\right)$, we get

$$
\Xi=\frac{P \cdot p_{1}}{P^{2}} q_{1}+\frac{P \cdot p_{2}}{P^{2}} q_{2}-\frac{P \cdot z}{P^{2}} y
$$

Formula (20) entails

$$
\Xi \cdot P=Q \cdot P
$$

In (19) the only quantity which is not a constant of the motion is $\left(P \cdot Q / P^{2}\right)$, it has the same dimension as $\tau_{1}, \tau_{2}$. In contrast setting 2 .

$$
T=\frac{P \cdot Q}{|P|}
$$

we give to $T$ the dimension of lenght (and time since $c=1$ ). One easily computes

$$
\left\{\frac{(J \cdot P)^{\alpha}}{P^{2}}, P_{\beta}\right\}=\Pi_{\beta}^{\alpha}
$$

whence we derive the relations

$$
\left\{\Xi^{\alpha}, P_{\beta}\right\}=\delta_{\beta}^{\alpha} \quad\left\{\Xi^{\alpha}, \frac{P^{2}}{2}\right\}=P^{\alpha}
$$

Owing to the constancy of $J_{\mu \nu}$ and $P_{\alpha}$, we can fix these quantities, say in particular

$$
\begin{array}{cc}
P_{\alpha}=k_{\alpha}, & \text { timelike vector } \\
\text { define } & k^{\alpha} k_{\alpha}=M^{2}
\end{array}
$$

Then we see that formula (19) defines the coordinates of a point which moves on a straight line when both $\tau_{1}, \tau_{2}$ run freely and independently from $-\infty$ to $+\infty$, and the direction of this line is given by $k^{\alpha}$.

\footnotetext{
${ }^{2}$ definition of $T$ has a different dimension in [15], but the same as here in [14] )
} 
Equation (19) becomes $\Xi^{\alpha}=$ Const. $+\left(\frac{P \cdot Q}{M}\right) \frac{k^{\alpha}}{M}$.

We can write $\frac{d \Xi^{\alpha}}{d T}=\frac{k^{\alpha}}{M}$ and consider respectively $T$ and $T / M$ as the proper time and the evolution parameter of the center of mass.

Similarly equations (23) supplemented by the trivial observation that $\left\{P, \frac{1}{2} P^{2}\right\}$ vanishes, can be viewed as canonical equations of motion for $\Xi$, generated by the one-body Hamiltonian $\frac{1}{2} P^{2}$.

Note however that in (19) the components of $\Xi$ do not commute among themselves; a similar situation was already encontered by Pryce [16].

From now on we shall focus on Unipotential Models characterized by the same interaction term for both particles, say $V_{1}=V_{2}=V$. Such models permit to write down explicit forms of the Hamiltonians. Moreover it seems that they are still general enough for dealing with most realistic interactions.

\section{Evolution of the canonical variables}

For the moment we postpone the physical interpretation; we are interested in the evolution of the canonical variables in terms of the parameters $\tau_{1}, \tau_{2}$, more generally we are concerned with statements that hold true regardless to the prescription used for solving the position equations (3). In this section, the only thing we assume about this prescription is Poincaré invariance.

At this stage the separation of external/internal variables is just formal and convenient for easy calculations. Because of Poincaré invariance and predictivity, $V$ can be only a function of the five independent scalars [13.

$$
P^{2}, \widetilde{z}^{2}, \widetilde{y}^{2}, \widetilde{z} \cdot \widetilde{y}, y \cdot P
$$

But in view of (14) it is more convenient for calculations to re-arrange them as

$$
P^{2}, \widetilde{z}^{2}, \widetilde{y}^{2}, \widetilde{z} \cdot \widetilde{y}, \frac{(y \cdot P)^{2}}{P^{2}}
$$

Unless otherwise specified we consider $V$ as a function of these five arguments. Soon it was observed [13] that

$$
\left(X_{1}-X_{2}\right) \widetilde{z}=\left(X_{1}-X_{2}\right) \widetilde{y}=0
$$

implying this

Proposition 1 In the motion, $\widetilde{z}$ and $\widetilde{y}$ will depend only on $\tau_{1}+\tau_{2}$. 
In contrast, $z \cdot P$ and $Q \cdot P$ will depend on both evolution parameters.

For any unipotential model, the individual energies $\frac{P \cdot p_{1}}{|P|}, \quad \frac{P \cdot p_{2}}{|P|}$ are separately conserved. Moreover the translation invariance of $V$ implies conservation of $P^{2}$, hence this obvious first integral

$$
N=H_{1}+H_{2}-\frac{\left(H_{1}-H_{2}\right)^{2}}{P^{2}}-\frac{P^{2}}{4}
$$

By elementary manipulations we find

$$
N=\widetilde{y}^{2}+2 V
$$

This important function defined on phase space is intimately related with the properties of relative motion; this can be intuitively seen as follows:

fixing numerical values to $H_{1}, H_{2}, P^{\alpha}$ (in particular $P \cdot P=k \cdot k=M^{2}$ ) results in a numerical value for $N$, let it be

$$
<N>=-\Lambda
$$

The quantum mechanical analog of this quantity appeared, denoted as $b^{2}$, in the work of Todorov [19.

Employing (for instance) the reduced mass of Galilean mechanics, say

$$
m_{0}=\frac{m_{1} m_{2}}{m_{1}+m_{2}}
$$

we can start from (27) and check that $\Lambda / 2 m_{0}$ is the leading term in the post-Galilean development of the quantity $M-\left(m_{1}+m_{2}\right)$ which is usually considered as binding energy for the bound states of relativistic quantum mechanic (see Appendix I).

In addition we shall see later on that $\frac{1}{2} N$ generates the evolution of the spatial relative canonical variables according to a one-parameter Hamiltonian scheme reminiscent of the nonrelativistic one-body mechanics, see equations (44) (45) below.

From (26) and with the notation (5) we can write

$$
\Lambda=\frac{M^{2}}{4}+\frac{\nu^{2}}{M^{2}}-\mu
$$

This important relation between $\Lambda$ and $M$ can be solved for $M^{2}$. We first write

$$
M^{4}-4(\mu+\Lambda) M^{2}+4 \nu^{2}=0
$$


From (28) it is already clear that $\Lambda+\mu>0$. But $M^{2}$ must be real and positive, so the possible values of $\Lambda$ are further restricted by the condition

$$
|\mu+\Lambda|>|\nu|
$$

which ensures that $M^{2}$ is real; under this condition we can write

$$
M^{2}=2(\mu+\Lambda) \pm 2 \sqrt{(\mu+\Lambda)^{2}-\nu^{2}}
$$

Moreover we must have at least one root of (29) positive. Since their product is non-negative these roots cannot have opposite signs, thus it will be sufficient to ensure that their sum is positive. Hence the condition

$$
\mu+\Lambda>0
$$

which ensures $M^{2}>0$. We can encompass both (30) and (32) by writting

$$
\mu+\Lambda>|\nu|
$$

Fortunately, the sign ambiguity in (31) can be removed, with help of the individual positive-energy condition (remind that $P \cdot p_{a} / M$ are the individual energies) that we assume henceforth

$$
P \cdot p_{1}>0, \quad P \cdot p_{2}>0
$$

Indeed, the numerical values of $P \cdot p_{1}$ and $P \cdot p_{2}$ are given by $\frac{M^{2}}{2} \pm \nu$. Requiring that both are strictly positive amounts to the condition

$$
M^{2}>2|\nu|
$$

Let $M^{\prime 2}, M^{\prime \prime 2}$ be the roots of equation (29). If both roots were to satisfy this inequality, it would contradict the equality $M^{\prime 2} M^{\prime \prime 2}=4 \nu^{2}$ implied by the last term in (29). It follows that

Proposition 2 Under the condition of positive individual energies, only one root of (29) is admissible

( Remark For strictly equal masses, only the plus sign may be taken in (31). Indeed this case means $\nu=0$, one root of equation (29) is obviously zero which must be rejected, and the other root is $M^{2}=$ $4(\mu+\Lambda)$ obtained from (31) by choosing the plus sign).

We can assume that $m_{1} \leq m_{2}$ without loss of generality. Thus $\nu \leq 0$, and (33) becomes

$$
\mu+\nu+\Lambda>0
$$


or equivalently

$$
m_{1}^{2}+\Lambda>0
$$

We see that either $\Lambda>0$ or it satisfies $-m_{1}^{2}<\Lambda<0$. In other words,

Proposition 3 Under the assumption of positive individual energies, either $\Lambda>0$ or $|\Lambda|<m_{1}^{2}$.

If we impose the individual energy conditions we additionally get $\frac{1}{2} M^{2}>$ $\nu$ (trivial) and also $\frac{1}{2} M^{2}>-\nu$, in other words

$$
M^{2}>m_{2}^{2}-m_{1}^{2}
$$

Thus, as soon as $m_{1} \neq m_{2}$, the collective mass of the whole system (lenght of linear momentum) cannot be arbitrarily small.

Let us check that taking the plus sign in (31) always yields an admissible root; eqn (35) implies that $\mu+\Lambda>-\nu$, thus looking at (31) we can write

$$
\frac{M^{2}}{2}>-\nu+\sqrt{(\mu+\Lambda)^{2}-\nu^{2}}>-\nu
$$

implying the individual energy condition (34). []

In view of the Proposition 2 above, taking the minus sign in (31) is excluded and we can write

$$
M^{2}=2(\mu+\Lambda)+2 \sqrt{(\mu+\Lambda)^{2}-\nu^{2}}
$$

Note that $M$ reduces to $m_{1}+m_{2}$ in the nonrelativistic limit, see Appendix I.

We now investigate the evolution of the dynamical variables. Let us first analyze the evolution of $\widetilde{z}$ and $\widetilde{y}$.

$$
\begin{aligned}
& \left(X_{1}+X_{2}\right) \widetilde{z}^{\alpha}=\left(X_{1}+X_{2}\right)_{\text {free }} \widetilde{z}^{\alpha}+2\left\{\widetilde{z}^{\alpha}, V\right\} \\
& \left(X_{1}+X_{2}\right) \widetilde{y}^{\alpha}=\left(X_{1}+X_{2}\right)_{\text {free }} \widetilde{y}^{\alpha}+2\left\{\widetilde{y}^{\alpha}, V\right\}
\end{aligned}
$$

where $V$ is a function of the quantities listed in (25).

Proposition 4 The Poisson brackets $\left\{\widetilde{z}^{\alpha}, V\right\}$ and $\left\{\widetilde{y}^{\alpha}, V\right\}$ are combinations of $\widetilde{z}^{\alpha}, \widetilde{y}^{\alpha}$ with coefficients that are functions of the five scalars listed in (25). 
Proof.

Consider first $\widetilde{z}^{\alpha}$. Obviously $\left\{\widetilde{z}^{\alpha}, P^{2}\right\}=\left\{\widetilde{z}^{\alpha}, \widetilde{z}^{2}\right\}=0$, and we also have that $\left\{\widetilde{z}^{\alpha}, y \cdot P\right\}=0$.

Then we compute

$$
\begin{gathered}
\left\{\widetilde{z}^{\alpha}, \widetilde{y}^{2}\right\}=2 \widetilde{y}^{\alpha} \\
\left\{\widetilde{z}^{\alpha}, \widetilde{z} \cdot \widetilde{y}\right\}=\widetilde{z}^{\alpha}
\end{gathered}
$$

hence

$$
\left\{\widetilde{z}^{\alpha}, V\right\}=2 \frac{\partial V}{\partial \widetilde{y}^{2}} \widetilde{y}^{\alpha}+\frac{\partial V}{\partial(\widetilde{z} \cdot \widetilde{y})} \widetilde{z}^{\alpha}
$$

Since $V$ is a function of the scalars (25) only, its partial derivatives involved in (41) obviously share this property.

Then consider $\widetilde{y}^{\alpha}$. Obviously

$$
\left\{\widetilde{y}^{\alpha}, P^{2}\right\}=\left\{\widetilde{y}^{\alpha}, \widetilde{y}^{2}\right\}=\left\{\widetilde{y}^{\alpha}, \frac{(y \cdot P)^{2}}{P^{2}}\right\}=0
$$

Then we compute

$$
\begin{gathered}
\left\{\widetilde{y}^{\alpha}, \widetilde{z}^{2}\right\}=-2 \widetilde{z}^{\alpha} \\
\left\{\widetilde{y}^{\alpha}, \widetilde{z} \cdot \widetilde{y}\right\}=-\widetilde{y}^{\alpha}
\end{gathered}
$$

hence

$$
\left\{\widetilde{y}^{\alpha}, V\right\}=-2 \frac{\partial V}{\partial \widetilde{z}^{2}} \widetilde{z}^{\alpha}-\frac{\partial V}{\partial(\widetilde{z} \cdot \widetilde{y})} \widetilde{y}^{\alpha}
$$

The partial derivatives involved in this formula are functions of the scalars (25).

In view of Prop. 1 it is convenient to set

$$
\lambda=\tau_{1}+\tau_{2}
$$

the equations of motion for $\widetilde{z}, \widetilde{y}$ are

$$
\begin{aligned}
& \frac{d \widetilde{z}}{d \lambda}=\left\{\widetilde{z}, \frac{1}{2} \widetilde{y}^{2}+V\right\} \\
& \frac{d \widetilde{y}}{d \lambda}=\left\{\widetilde{y}, \frac{1}{2} \widetilde{y}^{2}+V\right\}
\end{aligned}
$$

where the brackets can be computed as functions of $\widetilde{z}^{\mu}, \widetilde{y}^{\nu}$, and of the first integrals $P^{2}, y \cdot P$. Once $P^{2}$ and $y \cdot P$ have been fixed, the evolution of the spatial internal variables is given by a system of six first-order differential equations, to solve for six unknown functions; this problem has 
the structure of a nonrelativistic problem for one body in three dimensions. The four-vectors $\widetilde{z}$ and $\widetilde{y}$ remain within the 2-plane orthogonal to $k$ and to the (conserved) Pauli-Lubanski vector [13. Some solution

$$
\widetilde{z}=\zeta\left(\lambda, P^{2}, y \cdot P\right), \quad \widetilde{y}=\eta\left(\lambda, P^{2}, y \cdot P\right)
$$

of the system (44) (45) defines the evolution of the spatial relative canonical variables $\widetilde{z}, \widetilde{y}$. Interpretation in terms of world lines, relative positions and relative orbit will be given in the next section.

The collective evolution parameter $\lambda$ plays the role of the Newtonian time in the analogous one-body system. But in general $\lambda$ is not the time of any inertial observer. Therefore, in order to evaluate the schedule of the relative motion, we should express $\lambda$ in function of $T$ and insert the outcome into $\zeta$.

A complete knowledge of the motion also requires that we determine the evolution of $z \cdot P$ and $Q \cdot P$ in terms of $\tau_{1}, \tau_{2}$.

By sum and difference

$$
\begin{gathered}
\left(X_{1}+X_{2}\right) z \cdot P=2 y \cdot P+2\{z \cdot P, V\} \\
\left(X_{1}-X_{2}\right) z \cdot P=P^{2}
\end{gathered}
$$

We know that $V$ depends only on the five scalars (25) and we observe that $\left\{z \cdot P, \widetilde{y}_{\alpha}\right\}=0$, implying that $z \cdot P$ has a vanishing Poisson bracket with all scalars (25) except $\left.(y \cdot P)^{2} / P^{2}\right)$. We find $\{z \cdot P, y \cdot P\}=P^{2}$ hence finally

$\{z \cdot P, V\}$ only depends on the five scalars (25).

After integrating the system (44)(45) let us set

$$
\{z \cdot P, V\}=G\left(\lambda, M^{2}, \nu\right)
$$

We obtain

$$
\begin{gathered}
\left(X_{1}+X_{2}\right) z \cdot P=2 \nu+2 G\left(\lambda, M^{2}, \nu\right) \\
\left(X_{1}-X_{2}\right) z \cdot P=M^{2}
\end{gathered}
$$

Since

$$
\begin{gathered}
\left(X_{1}+X_{2}\right)=\frac{\partial}{\partial \tau_{1}}+\frac{\partial}{\partial \tau_{2}}=2 \frac{\partial}{\partial \lambda} \\
\left(X_{1}-X_{2}\right)=\frac{\partial}{\partial \tau_{1}}-\frac{\partial}{\partial \tau_{2}}=2 \frac{\partial}{\partial\left(\tau_{1}-\tau_{2}\right)}
\end{gathered}
$$

we finally have

$$
z \cdot P=\nu \lambda+\int G d \lambda+\frac{M^{2}}{2}\left(\tau_{1}-\tau_{2}\right)+\text { const. }
$$


Observing that

$$
\frac{M^{2}}{2} \pm \nu=P \cdot p_{1}\left(\operatorname{resp} . P \cdot p_{2}\right)
$$

we may write equivalently

$$
z \cdot P=\left(P \cdot p_{1}\right) \tau_{1}-\left(P \cdot p_{2}\right) \tau_{2}+\int G d \lambda+\text { const. }
$$

which reduces to eq. (3.6) of [13] when $G$ vanishes.

Similarly in view of (14) and (17) we can simply write (with $\partial$ according to (25) )

$$
\{Q \cdot P, V\}=\frac{\partial V}{\partial P^{2}}\left\{Q \cdot P, P^{2}\right\}
$$

where $\left\{Q \cdot P, P^{2}\right\}=2 P^{2}$ therefore

$$
\{Q \cdot P, V\}=2 P^{2} \frac{\partial V}{\partial P^{2}}
$$

which only depends on the five scalars (25).

After integration of the system (44) (45) let us set

$$
\{Q \cdot P, V\}=F\left(\lambda, M^{2}, \nu\right)
$$

in other words we perform the substitution

$$
F=\text { subs. }\left(\widetilde{z}=\zeta, \widetilde{y}=\eta, P^{2}=M^{2}, y \cdot P=\nu \mid \quad\{Q \cdot P, V\}\right)
$$

Now we can write

$$
\begin{gathered}
\left(X_{1}-X_{2}\right) Q \cdot P=\{Q \cdot P, y \cdot P\}=y \cdot P \\
\left(X_{1}+X_{2}\right) Q \cdot P=\left(X_{1}+X_{2}\right)_{\text {free }}+2\{Q \cdot P, V\} \\
\left(X_{1}+X_{2}\right) Q \cdot P=\frac{1}{2} P^{2}+2\{Q \cdot P, V\}
\end{gathered}
$$

Straightforward integration yields

$$
Q \cdot P=\frac{\nu}{2}\left(\tau_{1}-\tau_{2}\right)+\frac{M^{2}}{4} \lambda+\int F d \lambda+\text { const. }
$$

We see that modulo the solving of (44) (45) the evolution of $Q \cdot P$ in terms of $\tau_{1}, \tau_{2}$ will be given by a quadrature. In the very special case where $F \equiv 0$ (with our present notation) the above formula reduces to eq (3.8) of [13]. But most realistic potentials actually depend on $P^{2}$ which implies that $F$ differs from zero. 
In contrast $G$ vanishes in several cases of interest, for instance $G \equiv 0$ provided $V$ depends only on the dynamical variables $\widetilde{z}^{2}, P^{2}, L^{2}$. This statement stems from (16).

To summarize: After integrating (44) (45) we got $z \cdot P$ and $Q \cdot P$ as functions of $\tau_{1}, \tau_{2}$. Since we have the first integrals $P^{\alpha}=k^{\alpha}$ and $y \cdot P=\nu$ the only remaining dynamical variables to be determined are $\widetilde{Q}^{\beta}$. According to (18)

$$
\widetilde{Q}=\frac{z \cdot P}{P^{2}} \widetilde{y}-\frac{y \cdot P}{P^{2}} \widetilde{z}-\frac{\widetilde{P \cdot M}}{P^{2}}
$$

where the last term is also a first integral, thus everything in the righthand side is already detrmined. This observation achieves to determine the evolution of $q_{1}, q_{2}, p_{1}, p_{2}$, say

$$
q_{a}=\phi_{a}\left(\tau_{1}, \tau_{2}\right), \quad p_{b}=\psi_{b}\left(\tau_{1}, \tau_{2}\right),
$$

These functions define the two-dimensional "orbits" 3 of the evolution group in phase space.

\section{World lines in Unipotential Models}

The solutions of system (21) can be interpreted in terms of world lines provided we ultimately introduce the physical coordonates $x_{1}^{\alpha}, x_{2}^{\beta}$ as functions of the canonical coordonates. Our Cauchy surface for solving the position equations (3) is $(\Sigma)$ defined by $P \cdot z=0$, and our initial condition

$$
x_{a}^{\alpha}-q_{a}^{\alpha}=0 \quad \text { on the surface } \quad(\Sigma)
$$

can be formulated also as

$$
x_{a}^{\alpha}=q_{a}^{\alpha}+O(P \cdot z)
$$

where $O(P \cdot z)$ symbolically represents any expression which vanishes with $P \cdot z$. In principle formula (58) must be inserted into the solutions of (3) and yields the worldlines in terms of the individual evolution parameters, say $x_{1}\left(\tau_{1}\right), x_{2}\left(\tau_{2}\right)$.

The above prescription offers several advantages. First of all, setting

$$
r^{\alpha}=x_{1}^{\alpha}-x_{2}^{\alpha}
$$

\footnotetext{
${ }^{3}$ To avoid confusion with trajectories in space, we put the word orbit between quotation marks when it is meant in the group-theoretical sense.
} 
condition (59) implies that also $P \cdot r$ vanishes on $(\Sigma)$. In the rest frame we can write $x_{1}^{0}=x_{2}^{0}=T$, thus finally the manifold $(\Sigma)$ can be called the Equal-Time Surface .

At equal times the radius-vector $\widetilde{r}^{\alpha}$ moves on a curve that we may call the relative orbit. As observed long time ago [13, this curve lies on the 2-plane mentioned in the previous section (orbital plane).

This version of the Hamiltonian formalism clarifies the formal definition (20) written for the center of mass.

Indeed (20) is equivalent to (21), say

$$
\Xi=\frac{\left(P \cdot p_{1}\right) q_{1}+\left(P \cdot p_{2}\right) q_{2}}{P^{2}}+O(P \cdot z)
$$

In terms of the individual energies $M_{a}=\left(P \cdot p_{a}\right) /|P|$, we have

$$
\Xi=\frac{M_{1} q_{1}+M_{2} q_{2}}{M_{1}+M_{2}}+O(P \cdot z)
$$

Now at equal times $P \cdot z$ vanishes; fixing $P^{\alpha}=k^{\alpha}$ we can replace $q_{a}$ by $x_{a}$ and $P^{2}$ by $M^{2}$, so we are left with

$$
\left.\Xi\right|_{\Sigma}=\frac{M_{1} x_{1}+M_{2} x_{2}}{M_{1}+M_{2}}
$$

Notice that $M_{1}+M_{2}=M$ and remember that the individual energies reduce to the masses in the nonrelativistic limit (see Appendix II). In view of these remarks, formula (62) is more intuitive and significant when $P \cdot p_{1}$ and $P \cdot p_{2}$ are both positive: the analogy with the Newtonian definition of center of mass becomes obvious, which legitimates the positive-energy condition. At this stage it is clear that definition (20) agrees with the one proposed by Pryce [16]; similarly, formula (62) agrees with the notion of center of energy according to Fischbach et al [17.

\subsection{Rest-Frame description}

In practice, instead of trying to describe the motion in terms of the independent parameters $\tau_{1}, \tau_{1}$ we have better to fix the linear momentum $k^{\alpha}$, so defining a slicing of spacetime by the three-planes orthogonal to $k^{\alpha}$. These three-planes intersect both world-lines, which provides the rest-frame description of dynamics, as follows:

among all possible couples $x_{1}, x_{1}$ the slicing selects the equal-time configurations, characterized by $k \cdot r=0$. We just have to determine the sequence of these configurations, that is a one-parameter set. Picking up the equal-time configurations obviously induces a relation between 
$\tau_{1}$ and $\tau_{2}$, by cancellation of $P \cdot z$ in formula (49). One is left with a (possibly nonlinear) expression of $\tau_{1}-\tau_{2}$ as a function of $\lambda$.

After imposing (59), formula (49) permits us to express everything in terms of $\lambda$ only. Formula (20) permits to write

$$
\begin{aligned}
& q_{1}=\Xi-\left(\frac{\nu}{M^{2}}-\frac{1}{2}\right) z+O(P \cdot z) \\
& q_{2}=\Xi-\left(\frac{\nu}{M^{2}}+\frac{1}{2}\right) z+O(P \cdot z)
\end{aligned}
$$

But $z=\widetilde{z}+O(P \cdot z)$. Taking (59) into account (which implies $\widetilde{z}=$ $\widetilde{r}+O(P \cdot z))$, we put $P \cdot z$ equal to zero in (63) (64) and obtain the equal-time description of the motion

$$
\begin{aligned}
& x_{1}=\Xi-\left(\frac{\nu}{M^{2}}-\frac{1}{2}\right) \zeta\left(\lambda, M^{2}, \nu\right) \\
& x_{2}=\Xi-\left(\frac{\nu}{M^{2}}+\frac{1}{2}\right) \zeta\left(\lambda, M^{2}, \nu\right)
\end{aligned}
$$

These formulas yield a representation of both world lines in terms of the same parameter $\lambda$. But for the sake of a better understanding of the motion it is interesting to express $\lambda$ as a function of the center-of-mass proper time $T$.

Cancelling $P \cdot z$ in (49), our equal-time prescription implies

$$
\frac{1}{2} M^{2}\left(\tau_{1}-\tau_{2}\right)=-\nu \lambda-\int G d \lambda+\text { const. }
$$

Inserting into (57) yields the common value of $\Xi^{0}$ and $Q^{0}$ in the centerof-mass frame, say

$$
T=\lambda\left(\frac{M}{4}-\frac{\nu^{2}}{M^{3}}\right)-\frac{\nu}{M^{3}} \int G d \lambda+\frac{1}{M} \int F d \lambda+\text { const. }
$$

In principle this equation must be solved for $\lambda$. Let us stress that only in the very special case where $G$ and $F$ are constant, the time of the center of mass is for all orbits a linear function of the parameter $\lambda$. For instance, this situation is realized when both $\partial V / \partial P^{2}$ and $\partial V / \partial(y$. $P$ ) identically vanish, implying $G=F=0$. This situation will be referred to as the Academic Case.

Otherwise, in the most general case $\lambda$ and $T$ are related in a nonlinear way, but for exceptional orbits.

Note that for a physically admissible solution to (44) (45), $T$ should monotonously increase as a function of $\lambda$. In the academic case this 
is automatically ensured by the condition (34) of positive individual energies. When the interaction potential $V$ is more complicated we must demand $d T / d \lambda>0$, whereas we can write

$$
\frac{d T}{d \lambda}=\frac{M}{4}-\frac{\nu^{2}}{M^{3}}-\frac{\nu G}{M^{3}}+\frac{F}{M}
$$

For example assume for a moment that $G \equiv 0$, we are left with a simpler condition. If $F$ is positive no problem; otherwise the positive-energy condition must be replaced by a more restrictive and model-dependent condition (e.g. see the toy model of Section 6 ).

More generally, the discussion remains easy when $F$ and $G$ are bounded; as we shall see in the following section, this circumstance arises in case of circular motion.

\subsection{Circular Motion}

Circular motion is characterized by the constancy of $\widetilde{z}^{2}$, which implies that at equal times $\widetilde{r}^{2}$ also is constant. We can check that

Proposition 5 On any circular orbit the functions $G, F$ and the five dynamical variables (25) are constant.

Proof.

The interaction potential is a function of the five dynamical variables (24) We have four remarkable constants of the motion $P^{2}, N, y \cdot P$ and the square of angular momentum

$$
L^{2}=\widetilde{z}^{2} \widetilde{y}^{2}-(\widetilde{z} \cdot \widetilde{y})^{2}
$$

when fixed, they respectively take on the following numerical values

$$
M^{2},-\Lambda, \nu, l^{2}
$$

In the set (24) we can replace $\widetilde{z}^{2}, \widetilde{y}^{2}, \widetilde{z} \cdot \widetilde{y}$ by the equivalent set of scalars $\widetilde{z}^{2}, \widetilde{y}^{2}, L^{2}$. So let

$$
V=f\left(P^{2}, \widetilde{z}^{2}, \widetilde{y}^{2}, L^{2}, y \cdot P\right)
$$

Since $N=\widetilde{y}^{2}+2 V$ we can write

$$
N-\widetilde{y}^{2}=2 f\left(P^{2}, \widetilde{z}^{2}, \widetilde{y}^{2}, L^{2}, y \cdot P\right)
$$

This equation implicitly defines $\widetilde{y}^{2}$ as a function of $P^{2}, \widetilde{z}^{2}, N, L^{2}, y \cdot P$, if we leave apart a very exceptional case where $V$ linearly depends on $\widetilde{y}^{2}$ in a special manner (such case is not realistic anyway). 
Therefore it is sufficient that $\widetilde{z}^{2}=$ const. for having also $\widetilde{y}^{2}=$ const. , which in turn, according to (69), implies $\widetilde{z} \cdot \widetilde{y}=$ const..

Finally the five dynamical variables (24), or equivalently (25), remain constant on the circular orbit. []

It follows that $\lambda$ is a linear function of $T$ on circular orbits.

Theorem 1 If the interaction is such that $\{\widetilde{z}, V\}=0$ and $\frac{\partial V}{\partial(\widetilde{z} \cdot \widetilde{y})}=0$ there exist circular orbits; on these orbits the relative motion is periodic in terms of the center-of-mass time.

Proof. According to (44) we have that $\frac{d \widetilde{z}}{d \lambda}=\widetilde{y}$ and acording to (45) we get $\frac{d \widetilde{y}}{d \lambda}=-2 \frac{\partial V}{\partial \widetilde{z}^{2}} \widetilde{z}$. Equations (44) (45) are similar to that of a three-dimensional two-body problem. The case when $\left\{\widetilde{z}^{\alpha}, V\right\}$ is zero corresponds to the classical problem of motion under a central force, where circular orbits are known to exist.

As seen above, $\widetilde{y}^{2}$ is constant. In the analogy between our system and that of Galilean mechanics, $\lambda$ plays the role of time and $-\widetilde{y}^{2}$ represents the squared velocity. As well as a circular motion with a velocity of constant lenght is necessarily periodic in time, here we have that $\widetilde{z}$ and $\widetilde{y}$ are periodic functions of $\lambda$. Since we consider circular motion, $T$ is a linear function of $\lambda$ and vice versa, so periodicity in $\lambda$ implies periodicity in $T$. []

Example: any $V\left(P^{2}, \widetilde{z}^{2}\right)$ admits circular orbits.

\section{Extreme mass ratio, one-body limit}

We keep considering unipotential models. The case where one mass can be neglected in front of the other one is of practical interest when one tries to justify a resonable expression of $V$. Indeed it is naturally expected that in the limit of an extreme mass ratio we recover a system made of particle 1 moving in the external field created by particle 2 , the latter undergoing rectilinear uniform motion.

Without loss of generality we assume $m_{1} \ll m_{2}$. Note that we cannot just put $m_{2}$ to infinity. This can be seen already in the framework of Newtonian mechanics, because the gravitational potential created around particle 2 could not remain finite when $m_{2}$ tends to infinity. Therefore we shall rather put

$$
m_{1}=\gamma m_{2}
$$


and study the limit for $\gamma \rightarrow 0$.

In order to alleviate calculations, let us set $\varepsilon=\gamma^{2}$. We have

$$
\begin{aligned}
& \mu=\frac{1}{2} m_{2}^{2}(\varepsilon+1) \\
& \nu=\frac{1}{2} m_{2}^{2}(\varepsilon-1)
\end{aligned}
$$

whence we derive

$$
\begin{aligned}
\mu^{2}-\nu^{2}= & \frac{1}{4} m_{2}^{4}\left[(\varepsilon+1)^{2}-(\varepsilon-1)^{2}\right] \\
& \mu^{2}-\nu^{2}=\varepsilon m_{2}^{4}
\end{aligned}
$$

We whish to investigate whether, looking at things from the rest frame, the center of mass $\Xi$ and the spacetime position $x_{2}$ of the most heavy body actually coincide in the limit $\gamma \rightarrow 0$. By formula (20) we may write

$$
\Xi=Q+\left(\frac{y \cdot P}{P^{2}}\right) z-\left(\frac{P \cdot z}{P^{2}}\right) y
$$

where $Q=q_{2}+\frac{1}{2} z=q_{1}-\frac{1}{2} z$. On the mass shell we have that $P^{2}=M^{2}$ so

$$
\Xi=q_{2}+\left(\frac{1}{2}+\frac{\nu}{M^{2}}\right) z-\frac{P \cdot z}{M^{2}} y
$$

We can write $m_{1}^{2}=\varepsilon m_{2}^{2}$, where, of course $\varepsilon=\gamma^{2}$.

We are interested in what happens when $\gamma \rightarrow 0$.

In order to consider the most general case, let us set

$$
\Lambda=\alpha m_{2}^{2}
$$

without assuming for the moment any restriction about the magnitude 4 of $\alpha$.

In view of (70) and (72) we get

$$
\begin{gathered}
(\mu+\Lambda)^{2}-\nu^{2}=m_{2}^{2} \alpha(\Lambda+2 \mu)+\varepsilon m_{2}^{4} \\
(\mu+\Lambda)^{2}-\nu^{2}=m_{2}^{2} \alpha\left[m_{2}^{2} \alpha+m_{2}^{2}(\varepsilon+1)\right]+\varepsilon m_{2}^{4} \\
(\mu+\Lambda)^{2}-\nu^{2}=m_{2}^{4}(\alpha+1)(\alpha+\varepsilon)
\end{gathered}
$$

Notice that $2(\mu+\Lambda)=m_{2}^{2}(1+\varepsilon+2 \alpha)$. Inserting into (38) yields the rigorous formula

$$
M^{2}=m_{2}^{2}[1+2 \alpha+\varepsilon+2 \sqrt{(1+\alpha)(\alpha+\varepsilon)}]
$$

valid irrespective of the order of magnitude of $\alpha$ and $\varepsilon$. Now we are in a position to make the following statement

\footnotetext{
${ }^{4}$ Here we change the notation of $\left[14\right.$ by suppression of a factor $\frac{1}{2}$.
} 
Theorem 2 Provided we can neglect $\sqrt{|\Lambda|}$ in front of $m_{2}$, we have that $M^{2} \rightarrow-2 \nu$, which entails that, at equal times, $\Xi$ and $x_{2}$ coincide in the limit $\gamma \rightarrow 0$.

Indeed neglecting $\frac{\sqrt{|\Lambda|}}{m_{2}}$ amounts to cancel $\alpha$ in (77), that yields $-\frac{1}{2}$ as the limit of the ratio $\nu / M^{2}$, making the second term in the right-hand side of (74) to vanish; remember that the third term vanishes at equal times. []

Owing to Propo. 3 the condition for this result is always satisfied for negative $\Lambda$.

In contradistinction large positive values of $\Lambda$ forbid $\Xi$ to coincide with the heavy body in the limit of an extreme mass ratio. In this case $\alpha>0$ and formula (77) can be written

$$
M^{2}=m_{2}^{2}[1+2 \alpha+\varepsilon+2 \sqrt{(1+\alpha) \alpha} \quad \sqrt{1+\varepsilon / \alpha}]
$$

Define

$$
\beta=2 \alpha+2 \sqrt{\alpha^{2}+\alpha}
$$

Since $\alpha>0$ it is clear that $\beta>2 \alpha$. We get

$$
M^{2}=m_{2}^{2}(1+\beta)+O(\varepsilon)
$$

now using (71) yields

$$
\frac{1}{2}+\frac{\nu}{M^{2}}=\frac{\beta}{2(1+\beta)}+O(\varepsilon)
$$

which in general cannot vanish when $\varepsilon \rightarrow 0$. []

This situation can be physically interpreted as follows: Considered at equal times, our covariant definition of the center of mass $\Xi$ reduces to that of Fokker and Pryce [16]. See also Moeller [18]. Accordingly we notice that $\Xi$ is in fact a center of energy; therefore not only the masses but also the energies must be taken into account. Even if $m_{1}$ is very small, it must be understood that we cannot consider particle 1 as a test particle when its motion involves a too large amount of energy.

\section{Toy Model}

. Consider a harmonic potential

$$
V=\chi \sqrt{P^{2}} \widetilde{z}^{2}
$$


with $\chi$ a positive string constant (this potential differs from the one considered in [13] by because it is $P^{2}$-dependent, which alows for the correct dimension of the coupling constant).

The structure of the calculations derived from (81) is that of a nonrelativistic problem. Our notation is such that the relativistic potential and its nonrelativistic conterpart have opposite signs, so we have a positive $\Lambda$.

We must compute $F$, as defined in (52), according to (51) and after solving the reduced equations of motion (44) (45). The solution to this system is

$$
\begin{gathered}
\widetilde{z}=A \sin (\Omega \lambda+C)+B \cos (\Omega \lambda+C) \\
\widetilde{y}=A \Omega \cos (\Omega \lambda+C)-B \Omega \sin (\Omega \lambda+C)
\end{gathered}
$$

where $A, B$ are mutually orthogonal spacelike constant vectors (they span the orbital plane, their lenghts are the half-axes of an ellipse) and $C$ is a scalar constant; moreover we have

$$
\Omega=\sqrt{2 \chi|P|}
$$

Note that

$$
\{Q \cdot P, V\}=2 \frac{\partial V}{\partial P^{2}} P^{2}=\chi \sqrt{P^{2}} \widetilde{z}^{2}=V
$$

which is always negative. It is clear that $F$ will depend on $\lambda$ only through $\widetilde{z}^{2}$. Taking (82) into account and fixing $P^{\alpha}=k^{\alpha}$ we are left with

$$
\Omega=\sqrt{2 \chi M}
$$

whence we derive

$$
\begin{gathered}
<N>=2 \chi M\left(A^{2}+B^{2}\right)=-2 \chi M\left(a^{2}+b^{2}\right) \\
a^{2}+b^{2}=\frac{\Lambda}{2 \chi M}
\end{gathered}
$$

setting $A^{2}=-a^{2}, B^{2}=-b^{2}$. We obtain

$$
F=-\chi M\left[a^{2} \sin ^{2}(\Omega \lambda+C)+b^{2} \cos ^{2}(\Omega \lambda+C)\right]
$$

In order to calculate $\int F d \lambda$ we notice the primitive

$$
\begin{gathered}
\int^{\lambda}\left(a^{2} \sin ^{2}(\Omega \lambda+C)+\left(b^{2} \cos ^{2}(\Omega \lambda+C)\right) d \lambda=\right. \\
\frac{a^{2}+b^{2}}{2} \lambda+\frac{b^{2}-a^{2}}{2 \Omega} \sin (\Omega \lambda+C) \cos (\Omega \lambda+C)+\text { const. }
\end{gathered}
$$


Finally we find

$$
\int^{\lambda} F d \lambda=-\chi M\left[\frac{a^{2}+b^{2}}{2} \lambda+\frac{b^{2}-a^{2}}{4 \Omega} \sin (2 \Omega \lambda+2 C)\right]+\text { const. }
$$

so there is a secular (linear) term plus a periodic correction; in this particular example $\widetilde{r}$ is a periodic function of $\lambda$.

Since $z \cdot P$ has a vanishing bracket with $P^{2}$ and $\widetilde{z}^{2}$ it is obvious that $\{z \cdot P, V\}$ hence $G$, vanishes.

Owing to (34) we are sure that $\frac{M}{4}-\frac{\nu^{2}}{M^{3}}>0$. But the question is about $\frac{d T}{d \lambda}$. It can be directly read off (84) that

$$
|F| \leq \chi M\left(a^{2}+b^{2}\right)
$$

so the condition for $\frac{d T}{d \lambda}>0$ is

$$
\frac{M^{2}}{4}-\frac{\nu^{2}}{M^{2}}>|F|
$$

But

$$
|F| \leq \chi M\left(a^{2}+b^{2}\right)
$$

hence a sufficient condition

$$
\frac{M^{2}}{4}-\frac{\nu^{2}}{M^{2}}>\frac{\Lambda}{2}
$$

\section{Conclusion}

Center of mass and relative motion are well understood concepts for isolated two-body systems, provided interaction is of the unipotential type and physical positions are fixed by the equal-time prescription. A large part of our picture can be made abstractly but most physical features show up in the light of the equal-time description. The main scheme was put forward many years ago [7, but several important consequences are considered here for the first time.

In the present work we regard seriously the fact that the collective evolution parameter which arises in the reduced equations of motion (44) (45) is generally not a linear function of the center-of-mass time. This peculiarity (which doesnot concern the geometry of the orbit) automatically affects the schedule of relative motion.

This point led us to consider an important exception, the case of circular orbits, where the relative motion is periodic in $T$. 
Although the individual evolution parameters $\tau_{1}, \tau_{2}$ are generally not affine on the world lines, our equal-time treatment offers the possibility to end up with a description in terms of the center-of-mass time.

On the other hand, examinating the case of an extreme mass ratio provides an illustration of the nature of center of mass in relativity. Indeed the result expressed in Theorem 2 forces one to interprete $\Xi$ as a center of energy rather than of mass; this is in agreement with an ancient literature and with the spirit of relativity.

In this paper we considered relative motion essentially by analogy with a nonrelativistic one-body problem, naturally suggested by (44) (45). But of course the question as to know whether (and how) a ficticious relativistic one-body system can be invoked, is relevant and deserves a separate publication.

\section{APPENDIX}

I. Reverting from $\mu, \nu$ to $m_{1}, m_{2}$, formula (38) implies this useful approximation

$$
M=m_{1}+m_{2}+\frac{\Lambda}{2 m_{0} c^{2}}+O\left(1 / c^{4}\right)
$$

At first sight the appearence of $m_{0}$ in this formula seems to indicate that the non-relativistic expression for reduced mas goes over to the relativistic realm without modification; notice however that we could replace $m_{0}$ by any positive $m$ in this formula, provided that $m=m_{0}+O\left(1 / c^{2}\right)$. This remarks leaves open the possibility that the "good" relativistic generalization of the reduced mass may coincide with $m_{0}$ only in the nonrelativistic limit, as happens for instance with Todorov's [19] reduced $\operatorname{mass} m_{T}=\frac{m_{1} m_{2}}{M}$.

II. Defining $M_{a}=P \cdot p_{a} / M c^{2}$ we have

$$
M_{1}+M_{2}=M, \quad M_{1}-M_{2}=\frac{2 y \cdot P}{M c^{2}}=\frac{2 \nu}{M}
$$

But $2 \nu=\left(m_{1}^{2}-m_{2}^{2}\right) c^{2}$ thus $M_{1}-M_{2}=\frac{m_{1}^{2}-m_{2}^{2}}{M}$.

According to (86) we have $\frac{1}{M}=\frac{1}{m_{1}+m_{2}}+O\left(1 / c^{2}\right)$, so finally

$$
M_{1}-M_{2}=\frac{m_{1}^{2}-m_{2}^{2}}{m_{1}+m_{2}}+O\left(1 / c^{2}\right)=m_{1}-m_{2}+O\left(1 / c^{2}\right)
$$

Finally $M_{a}=m_{a}+O\left(1 / c^{2}\right)$. 


\section{References}

[1] J.A.Wheeler, R.P.Feynman, Rev. Mod. Phys. 17, 157 (1945).

[2] A.D. Fokker Zeitsch. f. Physik, 58, 386-393 (1929).

[3] P.A.M. Dirac, Forms of relativistic dynamics, Rev. Mod. Phys. 21, 392-9 (1949)

[4] D.G. Currie, Journ. Math. Phys. 4, 1470 (1963), Phys.Rev. 142, 817 (1966). D.G. Currie, T.F. Jordan, E.C.G. Sudarshan, Rev.Mod.Phys. 35, 350 (1963).

[5] Ph. Droz-Vincent, Lett. Nuov. Cim. 1839 (1969); Physica Scripta 2, 120 (1970)

[6] L. Bel, Ann. Inst. Henri Poincaré, 12 , 307 (1970).

R. Arens, Arch. for Rat. Mech. and Analysis, 47 , 255 (1972).

[7] Ph. Droz-Vincent, Reports in Math. Phys. 8, (1975) 79

[8] D. Dominici, J. Gomis, G. Longhi, Nuov. Cim. 48 A , 257 (1978);

Ibid. 48 B , 152 (1978); Ibid. 56 A , 263 (1980).

[9] I.T. Todorov, JINR Report E2-10125, unpublished (1976).

[10] D. J. Louis-Martinez, Phys. Letters B, 632, 733-739 (2006).

J.L. Friedman and Koji Uryu, Phys. Rev. D 73, 104039 (2006),

[11] D. Alba, L. Lusanna, M. Pauri, Jour. Math. Phys. 43 , 1677 (2002);

D. Alba, H. Crater, L. Lusanna, Jour. of Phys. A 40, 9585 (2007)

[12] L. Lusanna, Il Nuov. Cim. 65 B , 135 (1981).

[13] Ph. Droz-Vincent, Ann. Inst. H. Poincaré, 27, 407 (1977)

[14] Ph. Droz-Vincent, C. R. Acad. Sciences, Paris 290, 115 (1980)

[15] Ph. Droz-Vincent, J.M.Ph. (1996)

[16] Pryce, Proc. Roy. Soc. 195 A, 62 (1948)

[17] E. Fischbach, B.S. Freeman, W-K. Cheng, Phys. Rev.D, 23, 21572180, (1981); see Section 3, especially eq. (3.31 a ).

[18] C. Moeller Ann. Inst. Henri Poincaré, 11, 251 (1949).

[19] I.T. Todorov, in "Properties of fundamental interactions", 9 part C, A. Zichichi Ed. Editrice Compositori, Bologna (1973).

V.A. Rizov, I.T. Todorov, B. L. Aneva, Nucl. Phys. B 98, 447-471 (1975).

[20] H. Jallouli and H. Sazdjian, Ann. of Phys. 253, 376-426, (1997). 